\title{
The hybrid breakdown 1(t) locus induces interspecific hybrid breakdown between rice Oryza sativa cv. Koshihikari and its wild relative $O$. nivara
}

\author{
Kotaro Miura $^{\dagger 1,2)}$, Eiji Yamamoto ${ }^{\dagger 1)}$, Yoichi Morinaka ${ }^{3)}$, Tomonori Takashi ${ }^{3)}$, Hidemi Kitano $^{1)}$, \\ Makoto Matsuoka ${ }^{1)}$ and Motoyuki Ashikari*1) \\ 1) Bioscience and Biotechnology Center, Nagoya University, Furo-cho, Chikusa, Nagoya 464-8601, Japan \\ 2) Research Fellow of the Japan Society for the Promotion of Science, 8 Ichiban-cho, Chiyoda, Tokyo 102-8472, Japan \\ 3) Honda Research Institute Japan, Kazusa-Kamatari, Kisarazu, Chiba 292-0818, Japan
}

\begin{abstract}
Reproductive barriers are often observed in progenies derived from crosses between relatively distant species, such as cultivated varieties and their wild relatives. Such barriers hinder the transfer of agronomically important genes from one species to another. In the process of constructing a series of introgression lines (ILs) between Oryza sativa japonica cv. Koshihikari and wild species $O$. nivara, we observed the occurrence of hybrid breakdown, a reproductive barrier observed in $\mathrm{F}_{2}$ and later generations. Plants affected by this hybrid breakdown show extremely weak growth and die before heading. Linkage analysis and construction of ILs indicated that the locus $-h b d l(t)$, located on the short arm of chromosome 2 of $O$. nivara, induces hybrid breakdown in the Koshihikari genetic background. $h b d l(t)$ is identical to $h b d l$, which was previously identified in a cross between Koshihikari and Nona Bokra (indica) cultivars of $O$. sativa. We present genetic evidence that $h b d l(t)$ regulates hybrid breakdown and its location within $50 \mathrm{~kb}$. Possible reasons for this locus being responsible for hybrid breakdown in inter- and intraspecific crosses are discussed.
\end{abstract}

Key Words: rice, wild species, hybrid breakdown, reproductive barriers.

\section{Introduction}

The rice genus Oryza includes 22 species that are adapted to diverse ecological habitats (Vaughan et al. 2003). These species are classified into several complexes according to genome groups. Of the wild relatives, the AA genome group is the most accessible genetic resource, because cultivated $O$. sativa shares the same AA genome. Several studies have identified agriculturally desirable genes from wild species, including disease, and insect resistance, and some of these genes have been introduced into rice varieties (Brar and Khush 1997, Nguyen et al. 2003, Jena et al. 2006).

Interspecific hybridization is an important approach in rice breeding to transfer agronomically useful genes from wild relatives; however, in crosses between relatively distant species, such as cultivated varieties and wild relatives, there are often reproductive barriers that prevent gene flow between parental species. Reproductive barriers have been classified into several categories (Stebbins 1950). The first is geographical isolation, resulting in unique genotypes that are found in different environments. The second category includes prezygotic reproductive barriers that prevent the formation of hybrids. Such barriers include different flowering

Communicated by Darshan S. Brar

Received September 4, 2007. Accepted December 19, 2007.

*Corresponding author (e-mail: ashi@agr.nagoya-u.ac.jp)

$\dagger$ These authors contributed equally times and incompatible pollen tube growth between species. The last category includes postzygotic reproductive barriers, which act after hybrid formation and are classified into several subtypes. "Hybrid weakness (inviability)" and "hybrid sterility" are observed in $\mathrm{F}_{1}$ hybrids. When these traits are observed in $F_{2}$ or later generations, the phenomenon is termed "hybrid breakdown." Postzygotic reproductive barriers are serious problems in breeding programs because they prevent the exchange of genes between species even if hybrid formation is successful. Determining the mechanisms of these reproductive barriers will increase the chances of successfully introducing desirable agricultural genes from wild species.

We have constructed a series of introgression lines (ILs) from a cross between $O$. sativa cv. Koshihikari (japonica) and wild species $O$. nivara, which carries the AA genome, to identify agriculturally desirable genes and use them in a breeding program. In this process, we found several plants with extremely weak growth phenotypes. Because both parental and $F_{1}$ plants of these lines showed normal growth, the appearance of weak plants was a result of hybrid breakdown. In this study, we identified a locus on the short arm of chromosome 2 of $O$. nivara that induces hybrid breakdown in the Koshihikari genetic background. The locus could be identical to hybrid breakdown 1 ( $h b d l$ ), which Matsubara et al. (2007b) identified in progenies from a cross between $O$. sativa cv. Koshihikari (japonica) and $O$. sativa cv. Nona Bokra (indica). Here, we identified the locus from 
O. nivara in the Koshihikari genetic background that induced hybrid breakdown, and reasons for the locus responsible for hybrid breakdown in inter- and intraspecific crosses are discussed. High-resolution linkage and physical maps of the locus are also presented.

\section{Materials and Methods}

\section{Plant materials}

The rice varieties Koshihikari (Oryza sativa japonica) and $O$. nivara (accession number IRGC105444) and progenies from a cross between them were used. To create ILs, $\mathrm{F}_{1}$ plants (Koshihikari/O. nivara) were backcrossed with Koshihikari four times. ILs were selected from the $\mathrm{BC}_{4} \mathrm{~F}_{2}$ generation (Koshihikari/O. nivara/Koshihikari/Koshihikari/ Koshihikari/Koshihikari) using marker-assisted selection (MAS; Yano and Sasaki 1997, Yano 2001). Oryza nivara was kindly provided by the International Rice Research Institute (IRRI).

\section{Phenotypic evaluation}

Weak plants had strikingly fewer tillers compared to normal plants and exhibited weak root growth. Rice seeds were germinated in water in Petri dishes at $30^{\circ} \mathrm{C}$ for $72 \mathrm{~h}$ and then grown in a nursery for 1 month before transplanting in the field. To evaluate the occurrence of hybrid breakdown, data on plant height, root length and the numbers of tillers and roots were recorded.

\section{DNA extraction}

Genomic DNA was extracted from selected individuals of each $\mathrm{BC}_{2} \mathrm{~F}_{2}$ population and IL using the TPS method for linkage analysis and IL construction. For the TPS method, approximately $2-\mathrm{cm}$ lengths of rice leaf tips were harvested and ground using a Multi-Beads Shocker (Yasui Kikai, Osaka, Japan) in TPS buffer (100 mM Tris-HCl, pH 8.0, $1 \mathrm{M}$ $\mathrm{KCl}, 10 \mathrm{mM}$ EDTA). After centrifugation, the supernatant was recovered and an equal volume of isopropyl alcohol was added. Isopropyl alcohol-insoluble material was recovered by centrifugation, and the pellet was washed with $75 \%$ ethanol. The pellet was then dried and dissolved in TE $(10 \mathrm{mM}$ Tris-HCl, pH 8.0, 1 mM EDTA).

\section{Linkage analysis}

The purified DNA samples were genotyped using molecular markers. PCR-based markers, including simplesequence repeat (SSR) markers (McCouch et al. 2002, Ware et al. 2002, http://www.gramene.org/), cleaved amplified polymorphic sequence (CAPS) markers (Konieczny and Ausubel 1993), and single nucleotide polymorphisms (SNPs), identified by comparing the genomic DNA sequences of each parent, were used for linkage analysis and IL production. The sequences of primers used in this study are as follows: SNP2-10-U, GAGGGTAATAGGGCAT CAC; SNP2-10-L, GGGTGCACATGTATCTATCC; SNP212-U, TGCCCATGGCCATTCA; SNP2-12-L, GCAACCA
ACCCAGTGAAC; CH116-U, GCCTCAGATCAGGGAA TGAC; CH116-L, GCCTCAGATCAGGGAATGAC; C031-U, TATTGGGTTCCCTCCTCGG; C03-1-L, TGGCGGT CTAAGTGCCAA; dCAPS1-U, AACGAACGCACTCGA GTCTACTAGTACTACTCGTTAGAGA; dCAPS1-L, AG CTCCGCGTGGAGCAAGG; C03-2a-U, ATATGTATCCT GCATAAGCG; C03-2a-L, AATCGCAGAGAAAGGG; D12-1a-U, CGCTGAAACCTACTCATCCG; D12-1a-L, TGCTGCAGTGGGAAAGC; D12-2b-2-U, GGTGTTTTG AGTTCGACAGC; D12-2b-2-L，TCGACAAAGTGCAG CCC; D12-cJL-U, CGTCTCGTAGTTTACAGGCGG; and D12-cJL-L, AAGTGCCAAAAGGCTGTGTG. Markers S20351S and E5122S were released by the Rice Genome Project (http://rgp.dna.affrc.go.jp/). Genotyping was carried out using PCR according to Chen et al. (1997) or with the AcycloPrime-FP SNP Detection System (PerkinElmer, Wellesley, MA, USA) according to the manufacturer's protocol.

\section{Results}

Hybrid breakdown in progenies derived from a cross between $O$. sativa $c v$. Koshihikari and wild species, $O$. nivara

To identify agriculturally desirable genes in wild rice species, we have constructed a series of ILs, which are plant strains that possess relatively large segments of the donor parent's chromosomes with overlap in the recurrent parental background (Ashikari and Matsuoka et al. 2006). In the process of constructing ILs between $O$. sativa cv. Koshihikari and its wild relative $O$. nivara, we noted the appearance of plants with an extremely weak growth phenotype compared to their parental plants (Fig. 1). The weak plants showed short plant height, few tillers, and weak root growth, and died before heading. Because neither the parental plants nor $F_{1}$ plants showed such a phenotype, we concluded that the appearance of weak plants was the result of hybrid breakdown.

Hybrid breakdown is regulated by a recessive locus, $h b d 1(t)$

To analyze the genetic mechanism of this hybrid breakdown, we selected six weak plants from the $\mathrm{BC}_{2} \mathrm{~F}_{2}$ population and determined their chromosomal constitution using molecular markers covering the entire rice chromosome (Fig. 1). Although each of these weak plants had a unique chromosomal constitution, all possessed the $O$. nivara homozygote chromosome fragment on the short arm of chromosome 2 (Fig. 1C-H). These results suggest that the weak growth phenotype is controlled by a locus from $O$. nivara located on the short arm of chromosome 2.

A plant was isolated that carried a heterozygous chromosome segment on the short arm end of chromosome 2 in the Koshihikari genetic background from the $\mathrm{BC}_{4} \mathrm{~F}_{1}$ population (Fig. 2B). This plant showed no significant phenotypic differences from its recurrent parent, Koshihikari (Fig. 2A). Self-pollinated progenies of this plant included both normal and weak plants. The segregation of normal to weak plants 


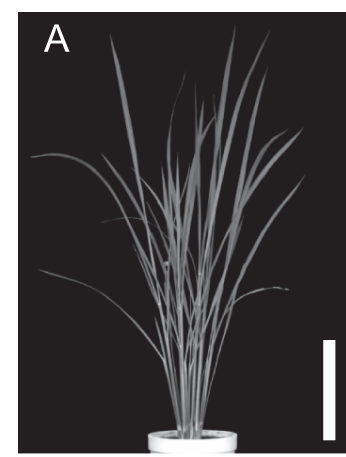

Koshihikari
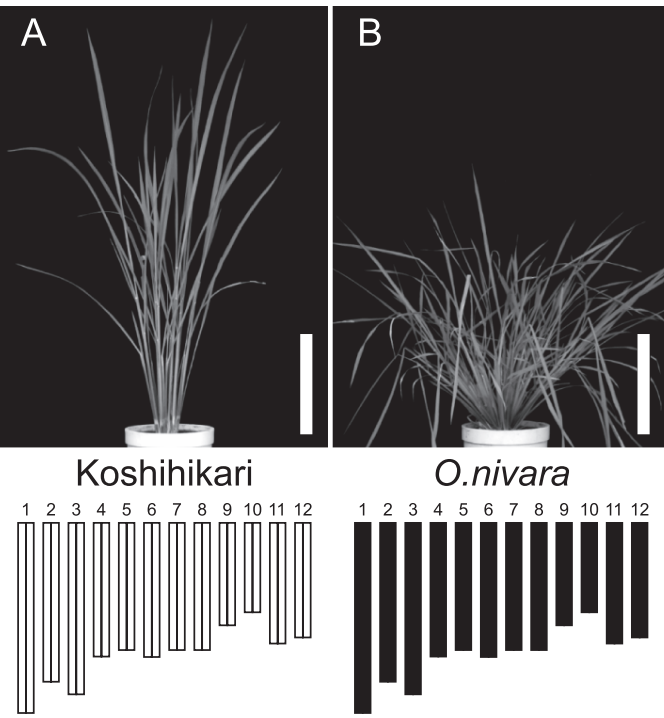

O.nivara

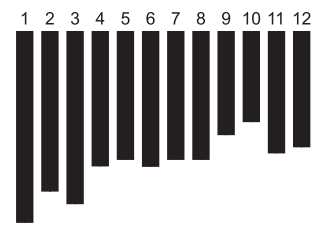

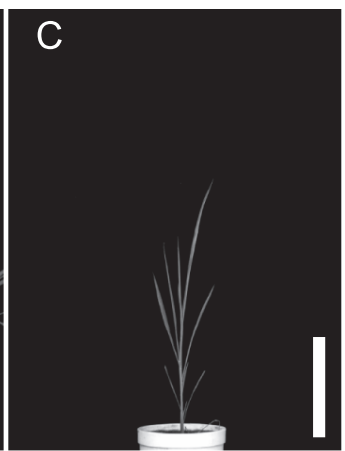

Weak plant 1

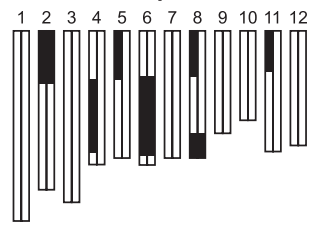

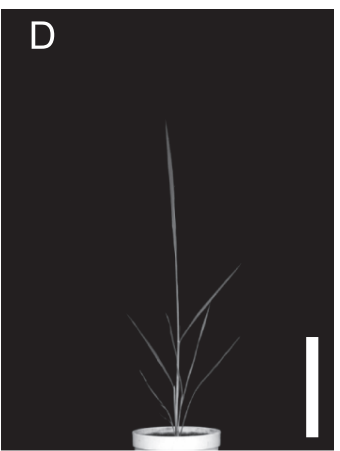

Weak plant 2

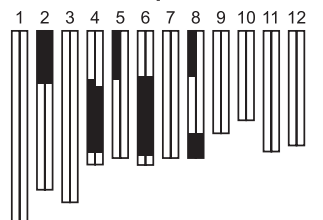

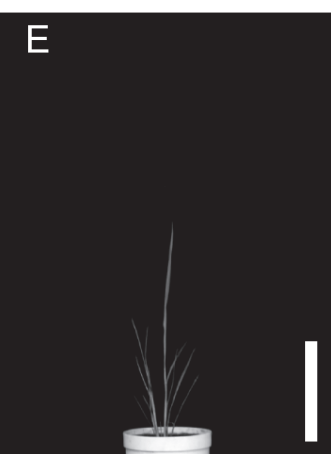

Weak plant 3

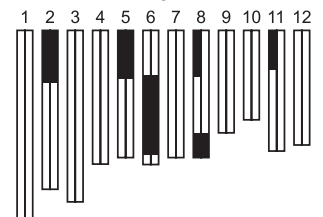

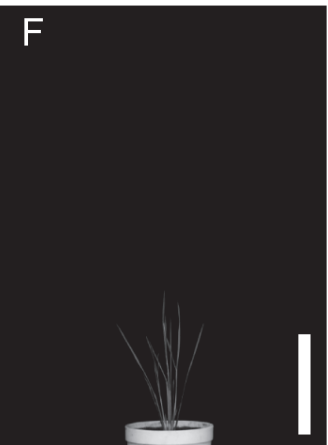

Weak plant 4

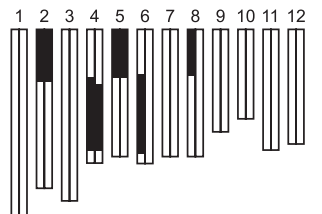

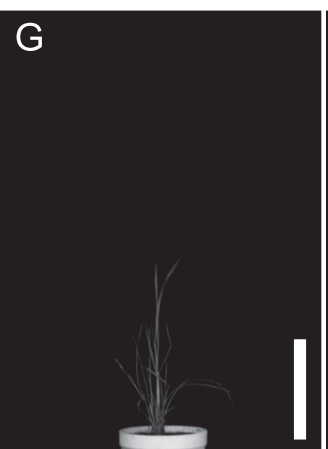

Weak plant 5

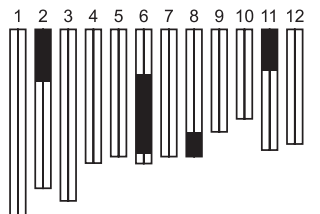

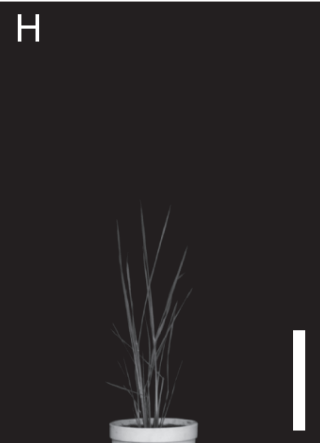

Weak plant 6

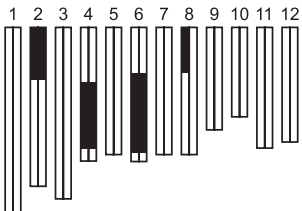

\section{[ Koshihikari chromosome \\ I O.nivara chromosome}

Fig. 1. Morphology and graphical genotype of parents and weak plants. Gross morphology and graphical genotypes of Koshihikari (A), O. nivara (B), and weak plants 1 to $6(\mathrm{C}-\mathrm{H})$. White bars in the gross morphology photos represent $20 \mathrm{~cm}$. White and black boxes in the graphical genotype panels indicate Koshihikari and $O$. nivara chromosome segments, respectively.

was $148: 44$, corresponding to a ratio of $3: 1\left(x^{2}=0.44\right)$. Thus, we concluded that in the Koshihikari genetic background, the weak phenotype is likely controlled by a single recessive locus on the short arm of chromosome 2 of $O$. nivara. Using this segregated population, we mapped this gene between molecular markers SNP-2-10 and SNP-2-12 within a 1.76-cM interval (Fig. 3). This chromosomal location overlaps with hybrid breakdown 1 ( hbdl), which is involved in hybrid breakdown between Koshihikari and Nona Bokra, indica cultivar (Matsubara et al. 2007b). This locus from Nona Bokra induces an extremely weak growth phenotype in the Koshihikari genetic background, and plants affected by this hybrid breakdown die before heading. The similar phenotype and chromosomal location suggest that the locus found in this study is identical to $h b d l$; however, since we do not have conclusive evidence that the identified locus is the same as $h b d l$, we named the locus hybrid breakdown 1(t), or $h b d 1(t)$. 


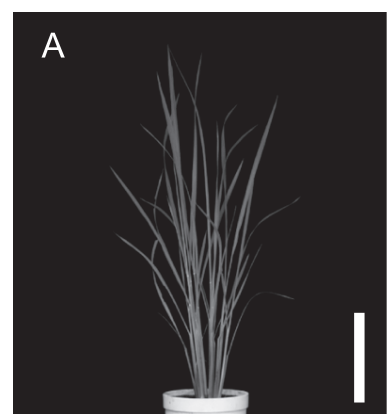

Koshihikari

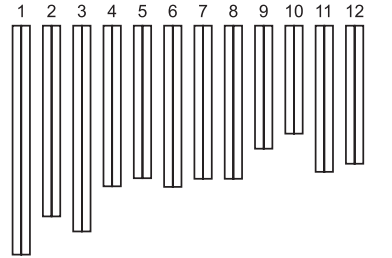

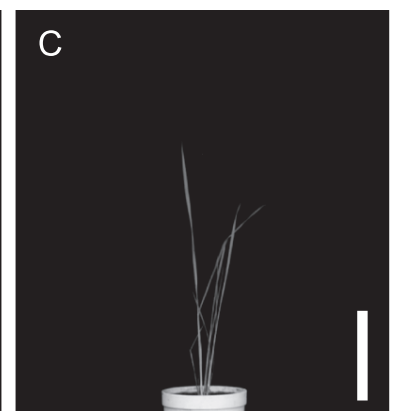

$h b d 1(t) / h b d 1(t)$

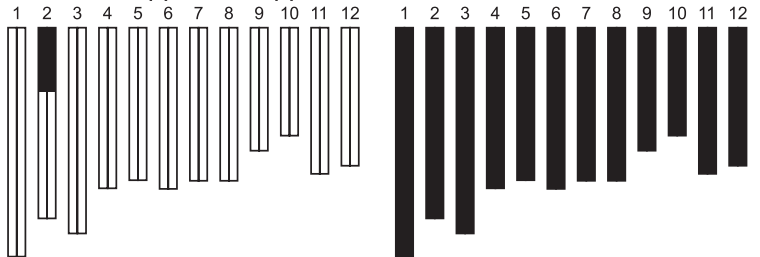

|| Koshihikari chromosome

I O.nivara chromosome
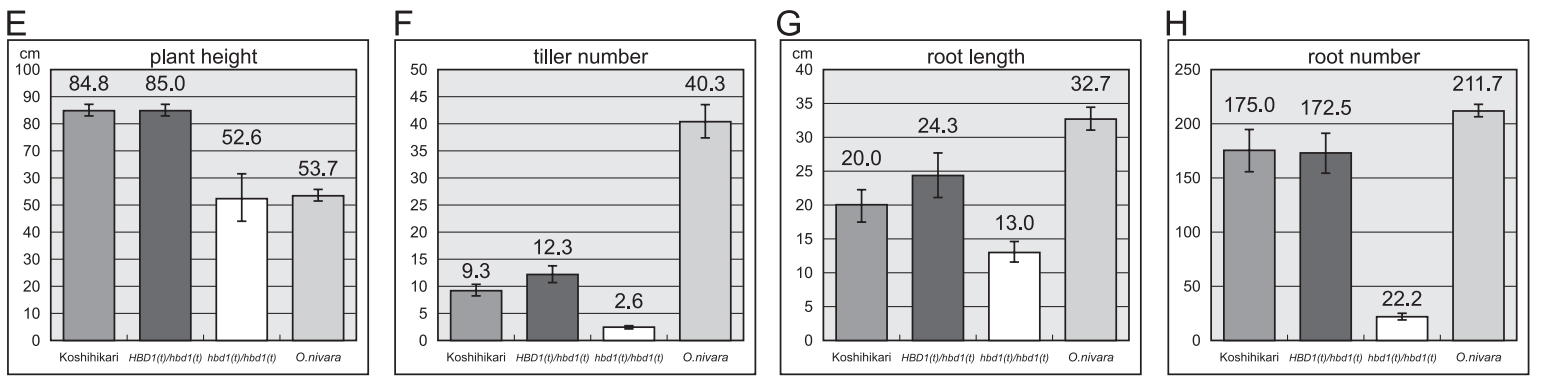

Fig. 2. Comparison of shoot and root traits in parents and weak plants. Gross morphology and graphical genotype of Koshihikari (A), an $H B D 1(t) / h b d l(t)$ plant (B), an $h b d 1(t) / h b d 1(t)$ plant (C), and $O$. nivara (D). White bars in the gross morphology photos represent $20 \mathrm{~cm}$. White and black boxes in the graphical genotype panels indicate Koshihikari and $O$. nivara chromosome segments, respectively. Comparisons of plant height $(\mathrm{E})$, tiller number $(\mathrm{F})$, root length $(\mathrm{G})$, and root number $(\mathrm{H})$. Values in $(\mathrm{E})-(\mathrm{H})$ are means with SDs $(\mathrm{n}=5$ plants).

\section{Phenotypic evaluation of hybrid breakdown with ILs}

ILs, which carry only a target chromosomal region in a unique genetic background, facilitate the precise evaluation of a locus of interest (Ashikari et al. 2005, Ashikari and Matsuoka 2006). To examine the effect of $h b d l(t)$ more precisely, we performed a screen for plants that were heterozygous [ $\operatorname{Hbdl}(t) / h b d l(t) ;$ Fig. 2B] and homozygous [hbdl(t)/ $h b d l(t)$; Fig. 2C] at the $h b d l(t)$ locus to compare the phenotype of their parental plants, Koshihikari (Fig. 2A) and O. nivara (Fig. 2D). Although heterozygous plants ( $\mathrm{Hbdll}$ $h b d l$ ) showed no significant differences from Koshihikari (Fig. 2A-B), homozygous plants ( $h b d l / h b d 1)$ showed significantly weaker growth (Fig. 2A and C). The IL ( $h b d l /$ $h b d l)$ showed the weak phenotype, with a plant height of $52.6 \mathrm{~cm}, 2.6$ tillers, a root length of $13 \mathrm{~cm}$, and 22 roots (Fig. 2E-F). These characteristics of the IL were all inferior to those of Koshihikari $(84.8 \mathrm{~cm}$ plant height, 9.3 tillers, $20 \mathrm{~cm}$ root length, and 175 roots). Furthermore, IL plants $[h b d l(t) / h b d l(t)]$ died before heading, which is consistent with the behavior of the $\mathrm{BC}_{2} \mathrm{~F}_{2}$ generation. Given that the IL showed all the characteristics of this hybrid breakdown, we concluded that a plant that is homozygous in $h b d l(t)$ from $O$. nivara is sufficient to induce hybrid breakdown in the Koshihikari genetic background.

\section{High-resolution mapping of $h b d 1(t)$}

As an initial step, we conducted high-resolution mapping using about 10,000 progenies from plants that were heterozygous at the $h b d l(t)$ locus (Fig. 2B). DNA was extracted at the seedling stage, and recombinant screening was conducted with molecular markers SNP-2-10 and SNP-2-12, which were used to determine the chromosomal location of $h b d l(t)$ (Fig. 3). In this step, 352 recombinant plants were obtained and used for further mapping. This mapping narrowed the $h b d l(t)$ candidate region to that between markers CH116 and D12-cJL, at $11.6 \mathrm{cM}$ on chromosome 2, with 18 recombinant plants (Fig. 4). The genotypes of the 18 recombinant plants were determined using these markers (Fig. 4). Although all plants showing a weak growth phenotype died before heading, progeny seeds of normal plants $\left(\mathrm{BC}_{4} \mathrm{~F}_{3}\right)$ were obtained, and their phenotypes were observed (Fig. 4). Comparison of the genotypes and phenotypes of 


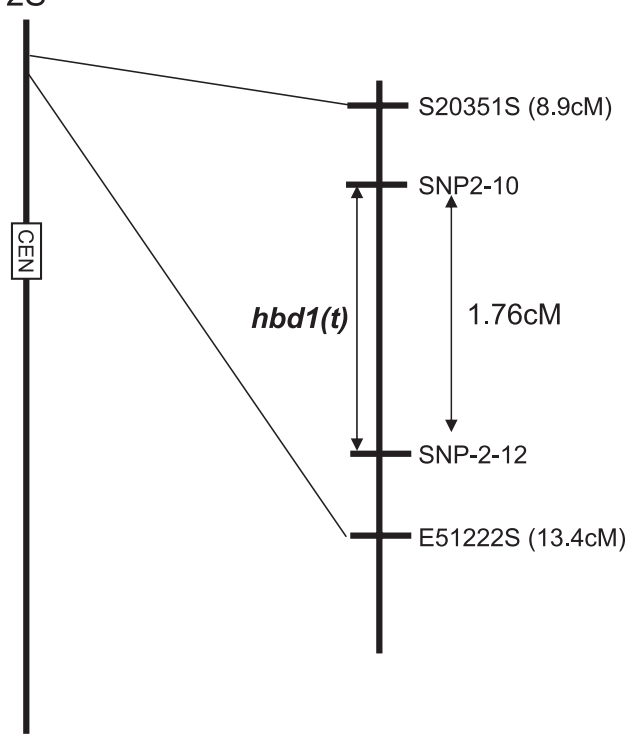

$2 \mathrm{~L}$

Fig. 3. Location of $h b d l(t)$ on chromosome 2. $h b d l(t)$ is located between molecular markers SNP2-10 and SNP-2-12. recombinant plants narrowed down the candidate region of $h b d 1(t)$ to between molecular markers dCAPS1 and D12-1a (Fig. 4). The region between these markers is about $50 \mathrm{~kb}$ in the Nipponbare genomic sequence.

To isolate $h b d l(t)$, it was necessary to construct a physical map of the candidate region. For this purpose, we generated BAC clone libraries of Koshihikari and $O$. nivara. To screen for BAC clones covering the candidate region for $h b d 1(t)$, we used molecular markers CH116, C03-1, dCAPS1, C03-2a, D12-1a, D12-2b-2, and D12-cj1. We obtained a BAC clone from the Koshihikari BAC library and a $\mathrm{BAC}$ clone from the $O$. nivara library that each cover the entire candidate region for $h b d l(t)$ (Fig. 4).

\section{Discussion}

In this study, we found several plants with an extremely weak growth phenotype in progenies of a cross between a japonica cultivar, Koshihikari, and the wild species, O. nivara (Fig. 1). Because both the parental and $\mathrm{F}_{1}$ plants showed normal growth phenotypes, we concluded that these weak plants were products of hybrid breakdown, a type of reproductive barrier.

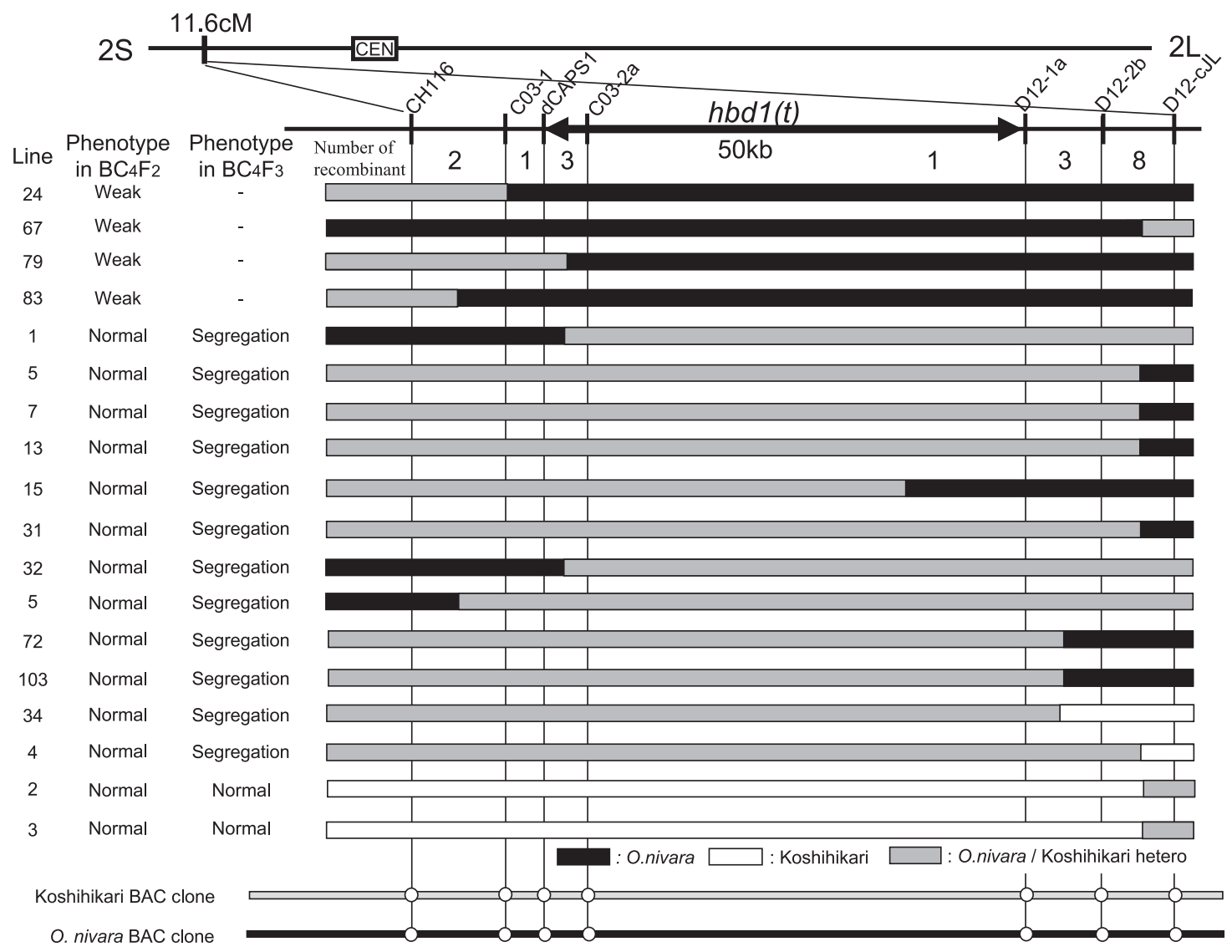

Fig. 4. High-resolution mapping and physical mapping of $h b d 1(t)$. Graphical genotype of 18 recombinants derived from a heterozygote of IL2-1. Black, white, and grey boxes in the graphical genotypes indicate chromosome segments of $O$. nivara, Koshihikari, and the heterozygote, respectively. The line numbers of the 18 recombinants and their phenotypes in $\mathrm{BC}_{4} \mathrm{~F}_{2}$ are indicated on the left, and the phenotypes of progenies $\left(\mathrm{BC}_{4} \mathrm{~F}_{3}\right)$ derived from normal $\mathrm{BC}_{4} \mathrm{~F}_{2}$ plants are also shown. Segregation was observed between weak and normal phenotypes. 
We found that a locus on the short arm of chromosome 2 of $O$. nivara induces hybrid breakdown in the Koshihikari genetic background. Previously, Matsubara et al. (2007b) reported that hybrid breakdown 1 ( $h b d 1)$, located on the short arm of chromosome 2 of indica cultivar, Nona Bokra, induces hybrid breakdown in the same genetic background with the same phenotype observed in this study. Further linkage analysis indicated that the candidate region of the locus identified in this study is included in the candidate region for $h b d 1$ (Matsubara et al. 2007b). The same phenotype and chromosomal location suggest that the locus identified in this study is the same as that identified by Matsubara et al. (2007b); therefore, we named the identified locus $O$. nivara $h b d 1(t)$. Recently, it was suggested that $O$. nivara may be the origin of O. sativa L. spp indica (Li et al. 2006). Furthermore, recent phylogenetic studies tend to support the independent origins of the indica and japonica rice cultivars ( $\mathrm{Zhu}$ and Ge 2005, Londo et al. 2006); therefore, it is possible that the $h b d l$ possessed by Nona Bokra is derived from $O$. nivara, reflecting the domestication steps of $O$. nivara to $O$. sativa L. spp indica. If so, this suggests that $h b d l(t)$ may be one of the determinants that distinguishes japonica and indica species, or one of the landmarks that enables us to trace the domestication steps of indica. This information will become clear when sequence information becomes available after cloning of $h b d l(t)$.

Many reports are available on the genetic analysis of hybrid breakdown in rice (Oka 1957, 1978, Oka and Doida 1962, Yokoo 1984, Sato and Morishima 1988, Wu et al. 1995, Li et al. 1997, Kubo and Yoshimura 2002, Fukuoka et al. 2005, Yamamoto et al. 2007, Matsubara et al. 2007a, 2007b). These studies demonstrated that hybrid breakdown is induced by the interaction of two or three genes derived from each parent. In this study, we identified one locus from the $O$. nivara chromosome, $h b d l(t)$, that is involved in hybrid breakdown between Koshihikari and $O$. nivara. Using plants that possess more complicated genetic backgrounds, we attempted to identify the locus or loci in the Koshihikari chromosome that interacts with $h b d l$ in the $O$. nivara chromosome to induce hybrid breakdown; however, we found no candidate locus for the interaction of two or three loci (data not shown). Thus, the genetic mechanism of this hybrid breakdown may be more complex.

Studies of reproductive barriers are useful for at least two reasons. First, they increase the chances of using diversity in breeding. Because one reproductive barrier is the prevention of gene flow by hybrid breakdown, an understanding of the mechanisms of such reproductive barriers could enable them to be avoided. Another value of such studies on reproductive barriers is in understanding biological mechanisms that distinguish species. Reproductive barriers are important to distinguish species and to maintain the identity of established species. Among the types of reproductive barriers, postzygotic reproductive barriers, such as hybrid breakdown, prevent gene exchange and reinforce genetic diversity among species. Several genes involved in postzygotic repro- ductive barriers have been isolated from animals (Wittbrodt et al. 1989, Ting et al. 1998, Brideau et al. 2006). Study of these genes has revealed tendencies in the development of postzygotic reproductive barriers: changes occur in amino acid sequences more than in regulatory regions, and positive selection is a driving force in the fixation of mutations. However, there have been no reports of the isolation of genes involved in postzygotic reproductive barriers in plants; therefore, it is unclear whether these tendencies are also present in plants. The high-resolution and physical maps in this study will be useful to isolate the gene involved in reproductive barriers and speciation, and will also help in understanding the molecular mechanism of such barriers.

\section{Acknowledgment}

We thank the International Rice Research Institute for providing rice materials.

\section{Literature Cited}

Ashikari, M., H. Sakakibara, S. Lin, T. Yamamoto, T. Takashi, A.Nishimura, E.R. Angeles, Q. Qian, H. Kitano and M.Matsuoka (2005) Cytokinin oxidase regulates rice grain production. Science 309: 741-745.

Ashikari,M. and M.Matsuoka (2006) Identification, isolation and pyramiding of quantitative trait loci for rice breeding. Trends Plant Sci. 11: $344-350$

Brar,D.S. and G.S.Khush (1997) Alien introgression in rice. Plant Mol. Biol. 35: 35-47.

Brideau, N.J., H.A. Flores, J.Wang, S. Maheshwari, X. Wang and D.A.Barbash (2006) Two Dobzhansky-Muller genes interact to cause hybrid lethality in Drosophila. Science 314: 1292-1295.

Chen,X., S.Temnykh, Y.Xu, Y.G.Cho and S.R.McCouch (1997) Development of a microsatellite framework map providing genome-wide coverage in rice (Oryza sativa L.). Theor. Appl. Genet. 95: 553-567.

Fukuoka,S., M.C.V.Newingham, M.Ishtaq, T.Nagamine, M.Kawase and K.Okuno (2005) Identification and mapping of two new loci for hybrid breakdown in cultivated rice. Rice Genet. Newslett. 22: 29.

Jena,K.K., J.U.Jeung, J.H.Lee, H.C.Choi and D.S.Brar (2006) Highresolution mapping of a new brown planthopper (BPH) resistance gene, Bph18(t), and marker-assisted selection for BPH resistance in rice (Oryza sativa L.). Theor. Appl. Genet. 112: 288-297.

Konieczny,A. and F.M.Ausubel (1993) A procedure for mapping Arabidopsis mutations using co-dominant ecotype-specific PCR-based markers. Plant J. 4: 403-410.

Kubo,T. and A.Yoshimura (2002) Genetic basis of hybrid breakdown in a japonica/indica cross of rice, Oryza sativa L. Theor. Appl. Genet. 105: 906-911.

Li,C., A.Zhou and T.Sang (2006) Genetic analysis of rice domestication syndrome with the wild annual species, Oryza nivara. New Phytol. 170: 185-194.

Li,Z., S.R.M.Pinson, A.H.Paterson, W.D.Park and J.W.Stancel (1997) Genetics of hybrid sterility and hybrid breakdown in an intersubspecific rice (Oryza sativa L.) population. Genetics 145: $1139-1148$. 
Londo, J.P., Y.C.Chiang, K.H.Hung, T.Y.Chiang and B.A.Schaal (2006) Phylogeography of Asian wild rice, Oryza rufipogon, reveals multiple independent domestications of cultivated rice, Oryza sativa. Proc. Natl. Acad. Sci. USA 103: 9578-9583.

Matsubara,K., T.Ando, T.Mizubayashi, S.Ito and M.Yano (2007a) Identification and linkage mapping of complementary recessive genes causing hybrid breakdown in an intraspecific rice cross. Theor. Appl. Genet. 115: 179-186.

Matsubara,K., S.Ito, Y.Nonoue, T.Ando and M.Yano (2007b) A novel gene for hybrid breakdown found in a cross between japonica and indica cultivars in rice. Rice Genet. Newslett. 23: 11-13.

McCouch,S.R., L.Teytelman, Y.Xu, K.B.Lobos, K.Clare, M.Walton, B.Fu, R.Maghirang, Z.Li, Y.Xing et al. (2002) Development and mapping of 2240 new SSR markers for rice (Oryza sativa L.). DNA Res. 9: 199-207.

Nguyen, B.D., D.S.Brar, B.C.Bui, T.V.Nguyen, L.N. Pham and H.T.Nguyen (2003) Identification and mapping of the QTL for aluminum tolerance introgressed from the new source, Oryza rufipogon Griff., into indica rice (Oryza sativa L.). Theor. Appl. Genet. 106: 583-593.

Oka,H.I. (1957) Phylogenetic differentiation of cultivated rice. XV. Complementary lethal genes in rice. Jpn. J. Genet. 32: 83-87.

Oka,H.I. and Y.Doida (1962) Phylogenetic differentiation of cultivated rice. XX. Analysis of the genetic basis of hybrid breakdown in rice. Jpn. J. Genet. 37: 24-35.

Oka,H.I. (1978) Phylogenetic differentiation of cultivated rice. XXI. The sporophytic pollen sterility: its genetic basis and interval relationship as shown by $F_{2}$ sterility. Jpn. J. Genet. 53: 397410.

Sato, Y.I. and H.Morishima (1988) Distribution of the genes causing $\mathrm{F}_{2}$ chlorosis in rice cultivars of the indica and japonica types. Theor. Appl. Genet. 75: 723-724.
Stebbins, G.L.Jr. (1950) Isolation and the origin of species. In: Stebbins, G.L. Jr. (ed.) Variation and Evolution in Plants. Columbia University Press, New York, pp. 189-250.

Ting,C.T., S.C.Tsaur, M.L.Wu and C.I.Wu (1998) A rapidly evolving homeobox at the site of a hybrid sterility gene. Science 282 : $1501-1504$.

Vaughan,D.A., H.Morishima and K.Kadowaki (2003) Diversity in the Oryza genus. Curr. Opin. Plant Biol. 6: 139-146.

Ware,D.H., P.Jaiswal, J.Ni, I.V.Yap, X.Pan, K.Y.Clark, L.Teytelman, S.C.Schmidt, W.Zhao, K.Chang et al. (2002) Gramene, a tool for grass genomics. Plant Physiol. 130: 1606-1613.

Wittbrodt,J., D.Adam, B.Malitschek, W.Maueler, F.Raulf, A.Telling, S.M.Robertson and M.Schartl (1989) Novel putative receptor tyrosine kinase encoded by the melanoma-inducing $T u$ locus in Xiphophorus. Nature 341: 415-421.

Wu,P., G.Zhang, N.Huang and J.K.Ladha (1995) Non-allelic interaction conditioning spikelet sterility in an $\mathrm{F}_{2}$ population of indica/ japonica cross in rice. Theor. Appl. Genet. 91: 825-829.

Yamamoto,E., T.Takashi, Y.Morinaka, S.Lin, H.Kitano, M.Matsuoka and M.Ashikari (2007) Interaction of two recessive genes, $h b d 2$ and $h b d 3$, induces hybrid breakdown in rice. Theor. Appl. Genet. 115:187-194.

Yano,M. and T.Sasaki (1997) Genetic and molecular dissection of quantitative traits in rice. Plant Mol. Biol. 35: 145-153.

Yano,M. (2001) Genetic and molecular dissection of naturally occurring variation. Curr. Opin. Plant Biol. 4: 130-135.

Yokoo,M. (1984) Female sterility in an indica-japonica cross of rice. Jpn. J. Breed. 34: 219-227.

Zhu, Q.H. and S.Ge (2005) Phylogenetic relationships among Agenome species of the genus Oryza revealed by intron sequences of four nuclear genes. New Phytol. 167: 249-265. 